\title{
Nasopharyngeal bursitis: from embryology to clinical presentation
}

This article was published in the following Dove Press journal:

International Journal of General Medicine

14 October 2010

Number of times this article has been viewed

\section{AE El-Shazly \\ $S$ Barriat \\ PP Lefebvre}

Department of Otorhinolaryngology and Head and Neck Surgery, Liege University Hospital, Liege, Belgium
Correspondence: AE El-Shazly Department of Otolaryngology, Rhinology Unit, Centre Hospitalier Universitaire de Liege, Domaine Universitaire du Sart Tilman B.35-B 4000 Liege I, Belgium Emailamrel_shazly@hotmail.com

\begin{abstract}
Nasopharyngeal bursitis is a relatively rare syndrome characterized by a collection of symptoms that multidisciplinary specialists should be aware of. Here we present an audit of cases presenting to a rhinology clinic over a two-year period, as well as an overview of the relevant embryology and different clinical presentations of nasopharyngeal bursitis. For 2008-2009, six patients were diagnosed to have nasopharyngeal bursitis, including four males and two females, of mean age 54 years. Two distinct pathologic types were observed, comprising three patients with classical Tornwaldt's cyst and three with crust-type bursitis. This audit highlights the importance of recognition of the crust-type of nasopharyngeal bursitis and its anatomic and clinical features. A combined endonasal and transoral endoscopic approach is a minimally invasive procedure and an effective method of treating both types of the disease. Our findings are discussed in relation to the embryology of the disorder, with a clinical emphasis on crust-type nasopharyngeal bursitis.
\end{abstract}

Keywords: nasopharyngeal bursitis, crust type, Tornwaldt's cyst, endoscopic disruption

\section{Introduction}

The nasopharyngeal bursa originates from the area of communication between the notochord and the foregut, ie, the pharyngeal endoderm. ${ }^{1,2}$ It is believed to be a remnant of embryonic communication between the notochord and the roof of the pharynx, that normally disappears during the second month of intrauterine fetal development. ${ }^{3}$ A Tornwaldt's cyst develops if the embryonic remnant becomes obstructed (cystic type), and if crusts adhere to the orifice without obstruction, this will form the crust type. ${ }^{4}$ Eagle $^{5}$ in 1939 indicated that in the crust type the orifice of the bursa is not obstructed despite crust formation. This crust sheds periodically from the nasopharynx, causing an offensive smell and unpleasant taste.

The cystic type of nasopharyngeal bursitis is more common and should form part of the differential diagnosis of nasopharyngeal abscesses and cysts. Although most patients are symptom-free, some will develop bursitis, a cyst, or an abscess. Therefore, the symptoms are of either a space-occupying lesion (nasal obstruction, eustachian tube obstruction with secretory otitis media, dysphagia, cranial nerve paralysis) or rhinitis (choanal discharge, halitosis, pharyngitis, laryngitis, bronchitis, gastritis). Being located at the posterior wall of the nasopharynx and extending toward the tubercle of the occipital bone, pulsating headache and occipital pain felt at the external occipital tuberculum is often reported.

The incidence of nasopharyngeal bursitis is approximately $4 \%$ in adults, but it can appear at any age. The peak incidence occurs between 15 and 30 years, with a Caucasian 
predominance and no gender predilection. ${ }^{5,6}$ Nonetheless, most of the published literature comprises selected case reports and describes the cystic type. No single report to the authors' knowledge has estimated the true incidence of crust-type nasopharyngeal bursitis or characterized its symptoms. In this clinicoanatomical audit, the aim was to identify the incidence of nasopharyngeal bursa disease, its different types, and its clinical presentation over two years in a single rhinology practice.

\section{Patients and methods Study design}

This was a two-year clinicoanatomical audit of nasopharyngeal bursitis at a rhinology practice in a university unit during 2008-2009. All patients diagnosed to have nasopharyngeal bursitis confirmed by endoscopic examination of the nasopharynx and computed tomography scan/magnetic resonance imaging were asked carefully about symptoms of Tornwaldt's syndrome. Correlation between symptoms and type of bursitis was noted (see Table 1). A surgical technique combining an endonasal and transoral approach in the form of disruption of the bursa with electrocauterization was performed to patients presenting with either the cyst type or the crust type. The patients were followed up for a minimum of 12 months.

Table I Clinical presentation of Nasopharyngeal bursitis

\begin{tabular}{ll}
\hline & Number of patients \\
\hline Sex & \\
Males & 4 \\
Females & 2 \\
Mean age & 54 \\
Type of bursitis & \\
Cyst & 3 \\
Crust & 3 \\
Symptoms & \\
Cyst & \\
$\quad$ Post nasal discharge & \\
$\quad$ Throat irritation & \\
$\quad$ Hemming & \\
Cough & \\
Crust & \\
Intermittent crust expectoration & \\
and retching & \\
Post nasal discharge & \\
Occipital pain & \\
\hline
\end{tabular}

Note: All patients had nothing significant in their past medical history apart from one patient with a pacemaker for atrial fibrillation who had cystic-type nasopharyngeal bursitis. No correlation could be made between occupation and the bursitis type because all six patients had different occupations

\section{Histologic analysis}

Paraffin embedding and tissue staining were performed using standard methodologies. Surgical samples of bursitis tissue were fixed in $10 \%$ formalin, and paraffin wax blocks were performed. Routine hematoxilyn staining of the nasal biopsy was then performed and specimens examined under low and high power fields. Representative photos from the slides were taken by a camera attached to software as shown in Figure 2.

\section{Results and discussion}

Results of the current audit (see Table) indicated that six patients (four males and two females, mean age 54 years) were diagnosed to have nasopharyngeal bursitis. All of the patients were Caucasian. The incidence was less than $1 \%$ of all rhinitis patients seen during the study period. Two distinct pathologic types were observed. For the cystic type, two patients with classical Tornwaldt's cyst and one with a fibrosed Tornwaldt's cyst were identified, while three patients with crust-type bursitis were diagnosed. The classical cyst type presented with postnasal discharge resulting in hemming, throat irritation, and cough, while the fibrosed type was discovered incidentally in a patient who complained of snoring. On the other hand, the crust type presented with crust expectoration and retching, with or without fetid postnasal discharge, and occasional occipital pain that lasts for a few days, with symptom-free intervals of a few days. For both types of disease, patients who underwent surgery using a combined endoscopic nasal and transoral approach had a complete recovery with no recurrence of symptoms at more than one year postoperatively (Figure 1c).

Interestingly, our audit showed an equal incidence of the cystic and the crust type of the disease. This highlights the importance of recognition of the crust type. Endoscopic examination of the nasopharynx after proper nasal decongestion will clearly show either a cyst or ulcer-like lesion covered with crusts, in the midline of the posterior wall of the nasopharynx (Figures 1a and 1b). Radiologic investigation (simple lateral view x-ray, computed tomography scan, and magnetic resonance imaging) is useful in showing adhesion of the bursa to the cervical vertebrae.

Although several surgical approaches with good outcomes have been described, ${ }^{6-9}$ we believe that the combined endoscopic endonasal and transoral approach with good electrocauterization is a minimally invasive and effective way of treating both types of nasopharyngeal bursitis (Figure 1c). 

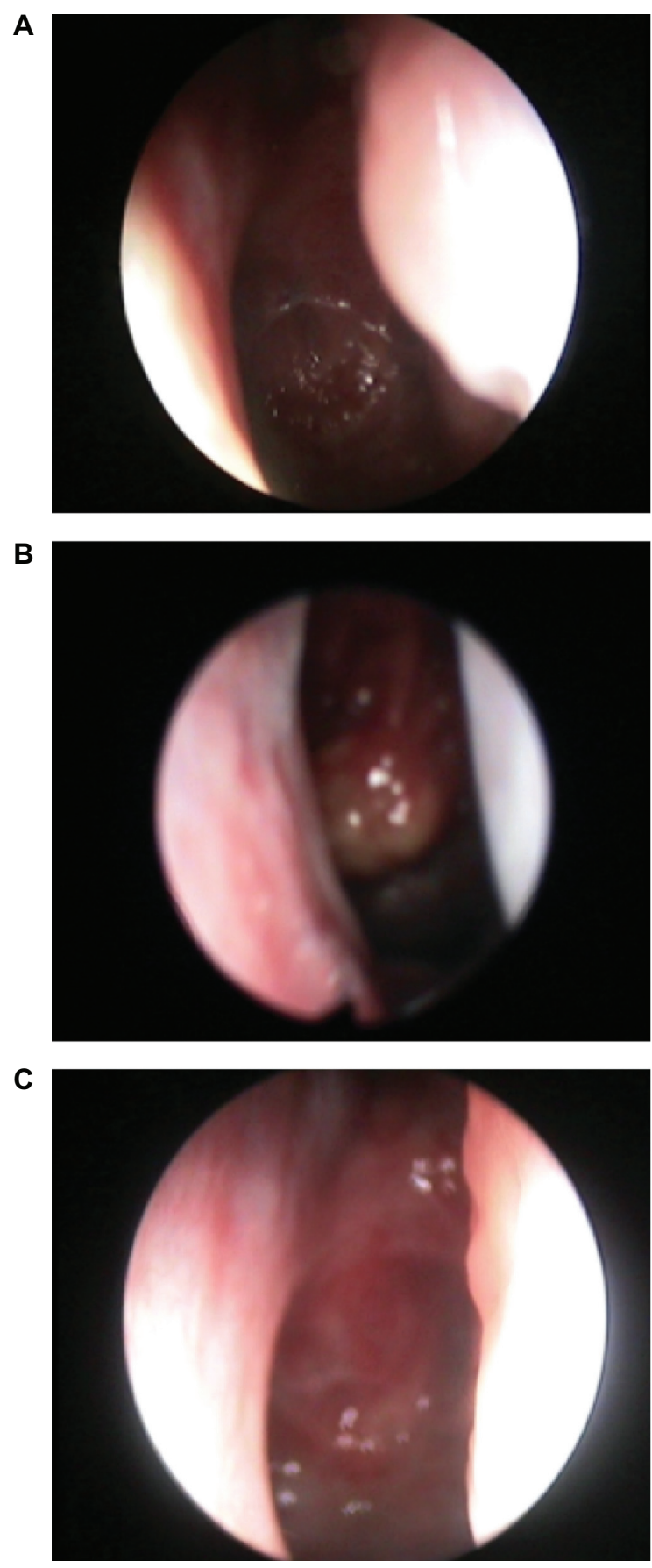

Figure I Thirty-degree rigid endoscopic appearance of nasopharyngeal bursitis. A) Crust type. Note the characteristic midline anatomic site with cicatricial streaks around the bursa. B) Cystic type. Photos are representative of three patients of each type showing very similar appearance. C) One-year postoperative endoscopic view of the crust type.

Nasopharyngeal bursa originate at the interface between the embryologic tissue from which the vertebrae develop. Notochord formation is an important change in the embryonic disc that takes place in the third week, and this is used by the embryo as a temporary axial skeleton. During migration of the intraembryonic mesoderm, the node of Hansen develops at the cephalic primitive streak, giving rise to the notochord process, in which a small central canal is formed. This canal connects the amniotic cavity and the yolk sac cavity. From this notochordal process, a rod-like solid definitive notochord becomes detached from the endoderm to lie in a position between the ectoderm and endoderm in the midline. This definitive notochord later becomes the permanent vertebral column. In approximately $3 \%-4 \%$ of embryos, an invaginated connection remains in the nasopharynx connecting the pharyngeal epithelium with the remnants of the notochord. This potential space allows migration of respiratory pharyngeal epithelial cells forming a nasopharyngeal bursa. Thus, the lesion is located in the middle of the posterior wall of the nasopharynx and extends to the tubercle of the occipital bone.

Tornwaldt's syndrome ${ }^{10}$ describes a group of symptoms resulting from inflammation, or cystic or abscess formation. In 1939, Eagle ${ }^{5}$ indicated that the orifice of the bursa is not obstructed in the crust type, despite the crust formation. This crust will shed from the nasopharynx, causing a bad smell and an unpleasant taste. In this audit, three patients presented with retching, expectoration of crusts, and/or fetid postnasal discharge and occipital pain, with no other described symptoms of nasopharyngeal bursitis. The crust reforms quickly, only to shed again every few days with completely symptom-free intervals in between.

Although most patients with nasopharyngeal bursa remain symptom-free, trauma to the nasopharynx in the form of nasal packing or adenoidectomy may result in obstruction of the bursa orifice and cyst formation. Interestingly, our patients developed their symptoms at an advanced age with no history of an initiating event, such as nasal packing or surgical trauma. Also, they remained completely symptomfree for years and presented suddenly with disturbing crusts of the nasopharynx. This indicates that the crust type could be a distinct variant of the disease that may develop at any time, and without recognized predisposing factors.

The cystic type of nasopharyngeal bursitis in our study was found to have two subtypes. One type is the classical cyst with postnasal discharge, throat irritation, and cough, while the other fibrosed type presented with irritation of the nasopharyngeal area and snoring.

Histopathologically, a Tornwaldt's cyst can be differentiated from a retention lymphoid cyst in that the former appears as an epithelial cyst lined with columnar epithelium on the surface ${ }^{11}$ while the latter shows lymphoid tissue only. 


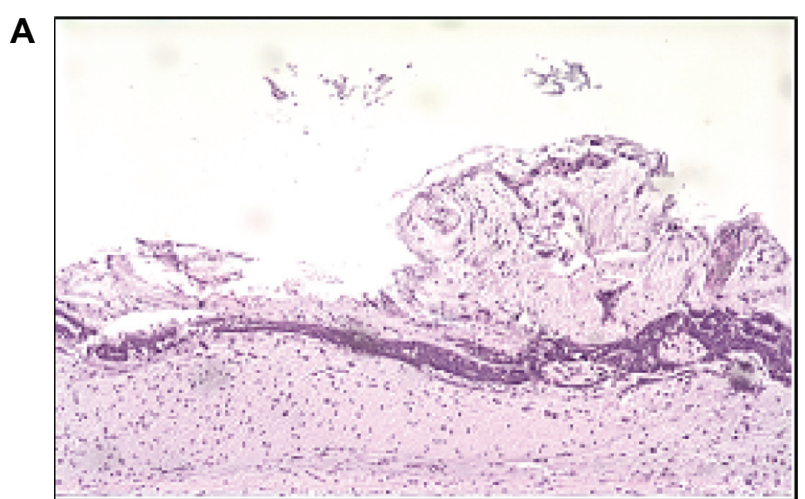

B

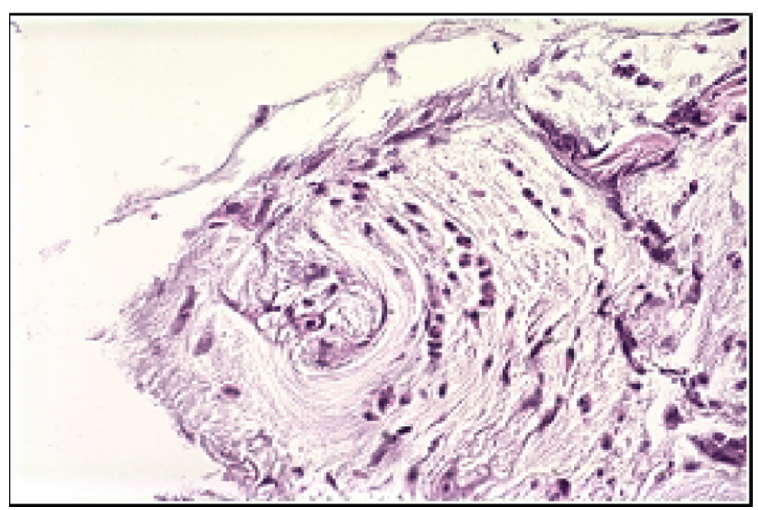

Figure 2 A) Low and B) high power fields showing histologic appearance of crust type bursitis showing reactive lymphoid mucosa with necrotic tissue.

However, the histologic picture of the crust type is not known. In Figure 2, we show the histologic appearance of the crust type that demonstrates reactive lymphoid mucosa with necrotic tissue. Interestingly, reactive lymphoid mucosa was also demonstrated in biopsies from the adjacent tissues, which may explain the cicatricial streaks around the bursa. This interesting histologic finding presents clinically as rapid formation of crusts with shedding and postnasal drip.

In conclusion, nasopharyngeal bursitis is a relatively rare congenital disease of the nasopharynx. The crust type is a recognized form of the disease and may produce no symptoms other than retching and irritative expectoration of crusts. Awareness of the crust type of nasopharyngeal bursitis would pick up many missed cases and increase appropriate referrals between generalists, pulmonologists, and otolaryngologists. Proper endoscopic nasopharyngeal examination with computed tomography and magnetic resonance imaging remains the best method of clinical assessment. Nasopharyngeal bursitis should be differentiated from nasopharyngeal carcinoma, which can mimic the ulcerative nature of nasopharyngeal bursitis, but usually does not form overlying crusts. The lack of symptoms, such as epistaxis and metastatic lymphadenopathy, as well as the characteristic midline anatomic site in the nasopharynx, are all in favor of the diagnosis of crust-type nasopharyngeal bursitis. Endoscopic interruption of the bursa with electrocauterization at the base is a minimally invasive technique that would suffice, and allows a better view of the operative field.

\section{Disclosure}

The authors report no conflicts of interest in this work.

\section{References}

1. Mayer FJC. Neue Untersuchungen aus dem Gebiete der Anatomie und Physiologie. Bonn. 1842.

2. Huber CC. On the relation of the chorda dorsalis to the anlage of the pharyngeal bursa or the median pharyngeal recess. Anat Rec. 1934;6:373-404.

3. Dorrance GM. The so-called bursa pharyngea in man. Arch Otolaryngol. 1931;13:187-224.

4. Kurihara H, Tanaka K, Yoshituru H. Tornwaldt's disease. Report of a case. J Otolaryngol Head Neck Surg. 1991;63:777-779.

5. Eagle WW. Pharyngeal bursa (Tornwaldt's bursa). Laryngoscope. 1939;25:199-207.

6. James J, MacMillan AS, Momose KJ. Tornwaldt's cyst. Br J Radiol. 1968;41:902-904.

7. Guggenheim P. Cysts of the nasopharynx. Laryngoscope. 1967;14: 2147-2168.

8. Kiernan DJ. Tornwaldt's syndrome. Arch Otolaryngol. 1963;77: 143-144.

9. Shaheen OH. Two cases of bilateral brachiogenic cysts of the nasopharynx. J Laryngol Otol. 1961;75:182-186.

10. Tornwaldt GL. Uber die Bedeutung der Bursa Pharyngea fur die Erkennung und Behandlung gewisser Nasenrachenraum-Krankheiten. Wiesbaden: Verlag von JF Bergmann. 1885.

11. Miyahara H, Mastunaga Ta, Hata N. Congenital disease of the epipharynx. JOHNS. 1990;6:1683-1691.
International Journal of General Medicine

\section{Publish your work in this journal}

The International Journal of General Medicine is an international, peer-reviewed open-access journal that focuses on general and internal medicine, pathogenesis, epidemiology, diagnosis, monitoring and treatment protocols. The journal is characterized by the rapid reporting of reviews, original research and clinical studies across all disease areas.

\section{Dovepress}

A key focus is the elucidation of disease processes and management protocols resulting in improved outcomes for the patient.The manuscript management system is completely online and includes a very quick and fair peer-review system. Visit http://www.dovepress.com/ testimonials.php to read real quotes from published authors. 